\title{
BRGÖ 2013
}

Beiträge zur Rechtsgeschichte Österreichs

\section{Vorwort}

Die Beiträge des vorliegenden Bands sind aus einer Tagung hervorgegangen, die im Rahmen eines vom österreichischen Wissenschaftsfonds FWF geförderten Projekts über Appellationen an den Reichshofrat vom 7. bis 9. September 2011 im Haus-, Hof- und Staatsarchiv in Wien durchgeführt wurde. Die Tagung verfolgte das Ziel, die Projektthematik in ihren rechtlichen und historischen Kontext einzuordnen, wobei insbesondere die Appellationstätigkeit anderer Gerichte auf dem Gebiet des Heiligen Römischen Reichs deutscher Nation berücksichtigt wurde. Damit soll zugleich an Forschungen der letzten Jahre über die Höchstgerichtsbarkeit in Europa angeschlossen werden. ${ }^{2}$

Die Perspektive des Projekts war dabei in drei Richtungen zu erweitern. Zum einen ging es darum, Appellationen am Reichshofrat auch über den chronologischen und thematischen Schwerpunkt des Projekts hinaus zu analysieren. Ein erheblicher Teil der Beiträge des Bands ist daher dem Reichshofrat gewidmet (Sellert, Franke, Kasper-Marienberg, Weitzel, Schnettger), wobei eine Form der am Reichshofrat üblichen Revision in die Betrachtung einbezogen wurde (Ortlieb).

Allerdings sollte der Reichshofrat, wie in der Forschung betont wurde, ${ }^{3}$ nicht isoliert betrachtet, sondern auf sein institutionelles und funktionelles Pendant-

\footnotetext{
1 "Appellationen an den Reichshofrat 1519-1740", FWF-Proj. Nr. P 20586-G08, Projektleitung: Hon.-Prof. Dr. Leopold Auer; Projektmitarbeiterin: Mag. Ellen Franke, [http://www.univie.ac.at/reichshofrat/index.php?article_id=24Eamp;clang=0/](abgerufen am: 28. 3. 2013).

${ }^{2}$ Hugo de SCHEPPER (Hg.), Höchste Gerichtsbarkeit im Spätmittelalter und der frühen Neuzeit (= Verzamelen en bewerken van de jurisprudentie van de Grote Raad NR 9, Amsterdam 1985); Bernhard DIESTELKAMP (Hg.), Oberste Gerichtsbarkeit und zentrale Gewalt im Europa der frühen Neuzeit (= Quellen und Forschungen zur höchsten Gerichtsbarkeit im Alten Reich 29, Köln-Weimar-Wien 1996); Leopold AUER, Werner OGRIS, Eva ORTLIEB (Hgg.), Höchstgerichte in Europa. Bausteine europäischer Rechtsordnungen (= Quellen und Forschungen zur höchsten Gerichtsbarkeit im Alten Reich 53, Köln-Weimar-Wien 2007); Rolf LIEBERWIRTH, Heiner LüCK (Hgg.), Akten des 36. Deutschen Rechtshistorikertages Halle an der Saale, 10.-14. September 2006 (BadenBaden 2008) 525-647; Ignacio CZEGUHN u.a. (Hgg.), Die Höchstgerichtsbarkeit im Zeitalter Karls V.: eine vergleichende Betrachtung (= Schriftenreihe des Zentrums für rechtswissenschaftliche Grundlagenforschung Würzburg 4, Baden-Baden 2011); zuletzt Alain Wijffels, C. H. (Remco) VAN RHEE (Hgg.), European Supreme Courts. A Portrait through History (London 2013).

${ }^{3}$ Siegrid WESTPHAL, Kaiserliche Rechtsprechung und herrschaftliche Stabilisierung. Reichsgerichtsbarkeit in den thüringischen Territorialstaaten 1648-1806 (= Quellen und
} 
das Reichskammergericht-bezogen werden. Auch bildeten Reichshofrat und Reichskammergericht nur ein Teilstück der höchstrichterlichen Rechtsprechung innerhalb des Reichs und in Europa. Die Perspektive war daher zum anderen um Beiträge zum Reichskammergericht (Schildt, Nehlsen-von Stryk) und zu anderen Gerichten, die für Appellationen zuständig waren, zu erweitern. Folie bildete dabei das Reich im weitesten Sinn: von den Niederlanden (Wijffels) über Böhmen (Kreuz) und Italien (Lepsius, Schnettger) bis zur Eidgenossenschaft (Lau). Reichsständische Höchstgerichte sind durch das Oberappellationsgericht in Celle repräsentiert (Stodolkowitz). Damit finden auch andere Gerichtsherren als "Kaiser und Reich" Berücksichtigung: Kommunen wie Bologna, Perugia, Florenz und Lucca (Lepsius), Reichsfürsten wie die Kurfürsten von Braunschweig-Lüneburg (Stodolkowitz) und die Erzherzöge von Österreich (Ortlieb), die Herrscher von Burgund (Wijffels) und Böhmen (Kreuz) sowie mit der Eidgenossenschaft ein republikanisches, jedenfalls bündisches Gebilde (Lau). Geographisch wird damit ein Raum angesprochen, der als europäisch zu bezeichnen ist-auch wenn er "Europa" bei weitem nicht ausschöpft.

Schließlich sollten zum dritten die rechtlichen Gegebenheiten beleuchtet werden, die die Appellationstätigkeit allgemein bestimmten. Dazu gehören insbesondere das für die Appellation besonders wichtige kanonische Recht (Becker) sowie die Appellationsprivilegien (Lück). Zu erweitern war die Perspektive auch in chronologischer Hinsicht in Richtung auf das Spätmittelalter (Becker, Lepsius).

Die im vorliegenden Band versammelten Beiträge beruhen auf einer vielfältigen Quellengrundlage: normative Texte, gelehrte und praxisorientierte Rechtsliteratur, Konsilien- und Spruchsammlungen (Becker, Lepsius, Lück, Nehlsen-von Stryk, Sellert, Wijffels, Stodolkowitz), Prozessakten (Lepsius, Franke, KasperMarienberg, Ortlieb, Wijffels, Kreuz, Stodolkowitz) und die sie verzeichnenden Inventare (Schildt, Sellert, Weitzel) sowie Instruktionen (Schnettger) und diplomatische Korrespondenzen (Lau). Der neueste Forschungsstand findet ebenso Berücksichtigung wie die Überlieferungssituation (Wijffels, Kreuz, Stodolkowitz). Auch methodisch gehen die Autorinnen und Autoren unterschiedliche Wege. Zur Interpretation von Rechtstexten und Rechtsliteratur (Becker, Lück, Nehlsen-von Stryk) sowie konfliktlösenden Praktiken (Lau) tritt die quantitative Analyse (Lepsius, Schildt, Sellert, Franke, Kasper-Marienberg, Ortlieb, Stodolkowitz) und die exemplarische Fallstudie (Franke) bzw. das Heranziehen von Beispielen aus der Praxis (Lepsius, Kasper-Marienberg, Weitzel, Ortlieb, Wijffels, Stodolkowitz). Auch ein kommunikationstheoretischer Ansatz wird verfolgt (Kasper-Marienberg). Der Band beleuchtet daher gleichermaßen Norm und Praxis der Appellation.

Forschungen zur höchsten Gerichtsbarkeit im Alten Reich 43, Köln-Weimar-Wien 2002) 6, 267. 
In dieser inhaltlichen und methodischen Vielfalt kristallisieren sich einige Grundfragen heraus, die in vielen Beiträgen des vorliegenden Bands angesprochen werden.

Dazu gehört prominent das Verfahrensrecht mit einer Reihe von immer wieder auftauchenden Fragen: verschiedene Formen der Appellation und ihre Abgrenzung voneinander und von anderen Rechtsmitteln bzw. Rechtsbehelfen (Judizial- und Extrajudzialappellation, processus ex eisdem actis und cum actoria, Vor- und Hauptverfahren, Nichtigkeitsklage, Revision); Zulässigkeitsvoraussetzungen und Rechtswirkungen (Devolutiveffekt, Suspensiveffekt); Formalia, Fristen und Kosten; das Vorbringen neuer Tatsachen und die Verfahrensdauer; Erfolgschancen, Urteilshäufigkeit und Missbrauch (Becker, Lepsius, Schildt, Nehlsen-von Stryk, Sellert, Franke, Kasper-Marienberg, Ortlieb, Wijffels, Stodolkowitz). Das wiederholte Auftreten dieser Phänomene ist natürlich kein Zufall, sondern wesentlich auf die Wirkung des römisch-kanonischen Prozessrechts zurückzuführen. Appellation und Revision stellen damit - wenig überraschend - die rechtsvereinheitlichende Wirkung der Rezeption unter Beweis. Die gemeinrechtliche Tradition konnte in Einzelfällen eine Eigendynamik entwickeln, die sich gegen reichsgesetzliche Regelungen fühlbar machte (Nehlsenvon Stryk). Trotzdem kam es nicht überall zu denselben Lösungen. Während sich an den Gerichten im Reich das Verbot der Appellation in peinlichen, also mit Leib- und Lebensstrafen bedrohten Strafsachen weitgehend durchgesetzt hatte, gehörten diese Agenden zu den wesentlichen - und forschungsgeschichtlich wichtigen - Aufgaben des Prager Appellationsgerichts (Kreuz). Während das Appellationsverfahren am Großen Rat in Mecheln in der Theorie ex eisdem actis durchgeführt werden sollte (Wijffels), war der erneute Parteienvortrag am Reichshofrat und anderen Gerichten ein wesentliches Merkmal des Appellation, das sie von der Revision unterschied. Die Praxis zeigt allerdings ein vielfältigeres Bild (Wijffels, Stodolkowitz). Eine markante Ausnahme von dieser Rezeptionstradition stellt die Eidgenossenschaft dar, in der sich eigenständige, an die Appellation erinnernde oder als solche bezeichnete Konfliktlösungsstrategien herausbildeten (Lau).

Über den Bereich des Prozessrechts hinaus geht die Problematik der Beschränkung der Appellation durch Appellationsprivilegien und der Appellationsbehinderung. Beide Phänomene betreffen die Verfassung und speziell die Gerichtsverfassung des Alten Reichs als Verhältnis zwischen Zentralgewalt und Territorien, das sowohl rechtlich gestaltet als auch praktisch austariert werden musste. Die Appellationsprivilegien haben sich dabei offenbar in Richtung auf eine Vereinheitlichung, auch Professionalisierung der Rechtsprechung ausgewirkt (Lück), während das Problem der Appellationsbehinderung eine insbesondere vom Reichshofrat mit wechselndem Erfolg bekämpfte Konstante der Rechtsprechung im Reich bildete (Weitzel). In diesen Zusammenhang gehören auch die Verwerfungen zwischen dem über Normtexte und Rechtsliteratur greifbaren Verfahrensrecht und der Prozesspraxis, die in einigen Beiträgen des Bands deutlich werden - Verwerfungen, die nicht immer politisch motiviert sein mussten 
(Lepsius) und auch als Handlungsspielräume gedeutet werden können (KasperMarienberg).

Auch darüber hinaus kam der Appellation ebenso wie der Revision politische und rechtspolitische Bedeutung zu. So hatte die Appellation beispielsweise wesentlichen Anteil an der Herausbildung von obersten Gerichten in den italienischen Kommunen des Spätmittelalters (Lepsius). In der Eidgenossenschaft avancierte die Zuständigkeit für Appellationen gelegentlich geradezu zum Definitionsmerkmal von Souveränität (Lau), in Böhmen sollte die Gründung des Appellationsgerichts nach der Niederwerfung eines Ständeaufstands die Zentralgewalt stärken (Kreuz). Ganz generell war die Kontrolle, die sich durch Appellation und Revision über die untergerichtliche Rechtsprechung ausüben ließ, nicht nur für die Parteien, sondern auch für den Gerichtsherrn von Interesse (Wijffels) - während man die Urteile des „eigenen“ Gerichts nicht mehr einer Appellation zu unterwerfen bereit war (Ortlieb). Im Fall Reichsitalien wurde die Zuständigkeit für Appellationen für den Reichshofrat zur eifersüchtig verteidigten Prärogative, die man sich auch durch praktische Überlegungen - die für regionale Lösungsmodelle gesprochen hätten - nicht entwinden lassen wollte. Interessenslagen der europäischen Diplomatie begrenzten den Handlungsspielraum ggf. beträchtlich (Schnettger). Für die Parteien konnte das Prozessieren vor Gericht im Sinne der "Justiznutzung" - wie im Fall der Frankfurter jüdischen Gemeinde - eine offenbar erstaunlich erfolgreiche Strategie sein, um wesentlich politische Positionen zu verteidigen (Kasper-Marienberg). Politische und rechtspolitische Überlegungen tragen dazu bei, die Bedeutung des Appellationsprozesses unabhängig von der (generell geringen) Anzahl von Endurteilen verständlich zu machen. Auch die Gerichte selbst entwickelten im ständigen Dilemma zwischen juristisch fundierter Kontrolle auf der einen und zügiger Rechtsprechung auf der anderen Seite in der Praxis eigene Lösungen (Lepsius, Wijffels, Stodolkowitz).

Was den Reichshofrat betrifft, reihen sich die hier vorgelegten Ergebnisse in den Forschungstrend der letzten Jahre ein. ${ }^{4}$ Auch im Zusammenhang mit Appellationen war der Reichshofrat nicht nur als Reichshöchstgericht tätig, sondern unterstützte den Kaiser generell bei seiner Aufgabe, über die Rechtsprechung im Reich zu wachen (Weitzel). Auch im Zusammenhang mit Appellation und Revision zeigt sich der Reichshofrat als eine prozessrechtlich außerordentlich flexible, stark auf gütliche Lösungen ausgerichtete Institution (Franke, KasperMarienberg, Ortlieb). Inwiefern dies die Bewertung der juristischen Qualität der Arbeit des Reichshofrats betrifft, erscheint derzeit allerdings als offene Forschungsfrage. Wenig Licht fällt im vorliegenden Band auch auf die von Gegnern des Kaisers und Teilen der insbesondere älteren Forschung stets unterstellte Rolle habsburgischer Interessen in der Arbeit des Reichshofrats.

\footnotetext{
${ }^{4}$ Wolfgang SELLERT, Vorwort, in: Die Akten des Kaiserlichen Reichshofrats Serie I: Alte Prager Akten, Bd. 1: A-D, bearb. von Eva ORTLIEB (Berlin 2009) 7-17.
} 
Überhaupt bleibt noch viel zu tun, um Appellation und Revision im Europa der Frühen Neuzeit systematisch zu analysieren. Zwei Ansätze erscheinen dafür besonders dringlich. Zum einen müsste der geographische Rahmen über das Reich hinaus auf andere Teile Europas ausgedehnt werden, wozu der begrenzte Rahmen der Tagung keinen Raum ließ. Zum anderen gilt für das Thema generell, was Matthias Schnettger in seinem Beitrag über Appellationen aus Reichsitalien feststellt: es wären viel mehr Fallstudien nötig, um Verfahrensdetails und Prozesspraxis und die sie prägenden Interessen aller Beteiligten besser verstehen zu können.

Die Herausgeber danken allen, die zum Gelingen der Tagung und zum vorliegenden Sammelband beigetragen haben: den Referentinnen und Referenten bzw. Autorinnen und Autoren, den Diskussionsleiterinnen und Diskussionsleitern (Univ.-Prof. Dr. Anja Amend-Traut, Würzburg; Univ.-Prof. Dr. Karl Härter, Frankfurt am Main; Univ.-Prof. Dr. Thomas Olechowksi, Wien; Univ.-

Prof. Dr. Thomas Simon, Wien; Univ.-Prof. Dr. Elmar Wadle, Saarbrücken; Univ.-Prof. Dr. Thomas Winkelbauer, Wien), Präsident Univ.-Prof. Dr. Dr.h.c. Clemens Jabloner sowie HR Dr. Sylvia Forster für einen eindrucksvollen Empfang im Verwaltungsgerichtshof, der Generaldirektion des Österreichischen Staatsarchivs sowie dem Direktor des Haus-, Hof- und Staatsarchivs Mag. Thomas Just und seinen Mitarbeiterinnen und Mitarbeitern, den Kolleginnen und Kollegen der Abt. KRGÖ des Instituts für Rechts- und Verfassungsgeschichte der Universität Wien (Katharina Bernold, Mag. Ellen Franke, DDr. Christoph Schmetterer, Mag. Kamila Staudigl-Ciechowicz) sowie der ÖAW für einen Reisekostenzuschuss. Bei den englischen Übersetzungen war die prompte und uneigennützige Hilfe von Univ.-Prof. Dr. Ronald Coons (University of Connecticut) für die Herausgeber von unschätzbarem Wert. Wir danken Univ.-Prof. Dr. Thomas Olechowski und den Herausgeberinnen und Herausgebern für die Aufnahme des Bands in die „Beiträge zur Rechtsgeschichte Österreichs". Außerdem gilt unser Dank dem Wissenschaftsfonds FWF, der die Durchführung der Tagung und die Drucklegung der Beiträge finanziert hat.

Der vorliegende Band erscheint zugleich elektronisch im Open-Access-Format: [http://hw.oeaw.ac.at/7232-5inhalt?frames=yes/]. Die damit verbundenen Möglichkeiten der Volltext-Recherche lassen den Verzicht auf Register hoffentlich vertretbar erscheinen. 
